Review Article

\title{
Mosquito-borne viral infections and diseases among persons and interfering with the vector activities
}

\begin{abstract}
Background: The main aspects of clinic manifestations, epidemiological data and case treatment about human arboviruses transmissible by mosquitoes are treated in this article. Mosquito-borne viral diseases also called arboviruses (for arthropod-borne viruses), can be transmitted to humans and animals by infected mosquitoes. Only certain types of mosquitoes can carry viruses and very few are even infected. A mosquito first becomes infected by feeding on a host that has the virus. Normally, the virus is transmitted between birds and mosquitoes, but occasionally certain types of mosquitoes can bite a person or animal. Arboviruses occur all over the world, but main viruses existing are dengue, yellow fever, West Nile virus, eastern equine encephalitis, western equine encephalitis, St. Louis encephalitis and La Crosse.
\end{abstract}

Results: Arboviruses human cases occur from spring to fall when mosquito populations are highest, peoples of all ages can get the disease, children are more likely to have severe illness or die, but persons over 50years of age have more severe disease. All mosquitoes do not have a virus in them, and the more mosquitoes bite to persons, the greater risk of catching a disease is there. Most peoples who get bitten by an infected mosquito never get sick, but rather just feel like they have the flu for a few days. In more serious cases, symptoms of mosquito-borne diseases occur 4 to 10days after being bitten by an infected mosquito. Illness can begin with fever, headache, muscle aches, nausea or vomiting, and may progress to seizures, paralysis, coma and possibly death. Persons who survive can suffer permanent health effects such as memory loss, paralysis or personality changes.

Conclusion: The most effective way to prevent mosquito-borne diseases is to prevent being bitten by mosquitoes. Always take personal protective measures to avoid mosquito bites, especially when mosquito-borne viruses have been identified near habitation. Being aware of mosquito and mosquito-borne disease activities in the area, it can allow taking action to protect ourself and others. When anybody has a mosquito-borne disease and gets any flulike symptoms especially in the summer months, it is necessary to see physician at once. Integrated mosquito management approaches should be adopted for getting an effective and efficient control of vectors that starts from identification of the species of mosquito causing the problem to the biological, environmental and chemical categories for controlling the target species.

Keywords: mosquito disease, vector control, vector monitoring, vector surveys, arboviruses, encephalitis
Volume 3 Issue 2 - 2016

Muhammad Sarwar

Nuclear Institute for Food \& Agriculture, Pakistan

Correspondence: Muhammad Sarwar, Nuclear Institute for Food \& Agriculture (NIFA), Tarnab, Peshawar, Pakistan, Email drmsarwar64@yahoo.com

Received: November 01, 2016 | Published: December 28, 2016

\section{Introduction}

Several insect-borne viruses circulate on the planet each year and are capable of causing disease in humans and other animals. The most common mosquito-borne viruses include West Nile virus, Eastern equine encephalitis virus and La Crosse virus, in addition Saint Louis encephalitis virus has also been detected in the past. Mosquito-borne viruses are most active in late spring through early fall, and can infect birds, horses and other animals in addition to humans. If public health reports are positive for birds or animals in an area, or if large numbers of mosquitoes are seen, there could be at increased risk of infection. Thirty four types of arboviruses from 183 types have been isolated and associated with human diseases. Four of them are important in public health and are involved with epidemics; they are namely, dengue, yellow fever, oropouche and encephalitis viruses. Dengue is associated with human epidemic diseases in urban areas while yellow fever in rural areas. Basically, oropouche causes a febrile disease, sometimes accompanied with aseptic meningitis, and dengue is associated with rash febrile disease, while yellow fever determines hemorrhagic fever. Thirty other arboviruses are involved with febrile illnesses in a few and sporadic cases. All arboviruses (apart from dengue) are maintained within a sylvatic cycle in the forest, where several species of haematophagous insects act as vectors and wild vertebrates are involved as hosts. Dengue has a cycle where the Aedes aegypti mosquito is the vector and man is the host. With the exception of the four viruses associated with epidemics which determine great economic and social impacts, including death (as in the case of yellow fever), the real involvement of these viruses as systematic agents of human disease is unknown. Further studies are needed to clarify unclear aspects of the epidemiological cycles of these viruses. ${ }^{1-3}$

\section{Role of mosquitoes in transmission of viruses}

Viral diseases are one of the most severe clinical manifestations of arbovirus infection and may result in death or leave severe consequences in survivors, wherein mosquitoes represent an important public and veterinary health problems.

\section{Zika virus}

Zika virus is transmitted to humans via a bite by an infected $A$. aegypti mosquito. Another mosquito similar to A. aegypti, A. albopictus, also has the potential to transmit Zika virus. 
An Aedes mosquito becomes infected with Zika virus when it bites a sick person who has the virus in his blood, that mosquito can then pass the virus on to other peoples. Mosquitoes remain infectious for life and can infect several peoples. Typical symptoms may include fever, headache, sometimes with pain behind the eyes, muscle and joint pain, red rash and conjunctivitis. The illness is self-limiting, lasting four to seven days and is diagnosed by having a blood test. There is no vaccine to protect against $\mathrm{Zika}$ virus that is an emerging mosquitoborne disease globally and it has been diagnosed in travelers from affected countries. The same mosquitoes that transmit dengue and chikungunya also transmit Zika virus.,

\section{Dengue fever}

Dengue is a viral disease transmitted from person to person by mosquitoes, it is usually an acute, nonfatal disease, characterized by sudden onset of fever, headache, backache, joint pains, nausea and vomiting. While most infections result in a mild illness, some may cause the severe forms of the disease. Dengue hemorrhagic fever, for example, is characterized by severe rash, nosebleeds, gastrointestinal bleeding and circulatory failure resulting in dengue shock syndrome and even death. In dengue infected mosquitoes Aedes aegypti or Aedes albopictus, the virus is present in the salivary glands of the mosquito. When a female Aedes bites a human for food, it injects saliva into the wound where the anti-coagulants contained in its saliva facilitate feeding. Without knowing, the mosquito also injects the dengue virus into the host. Since the virus can be passed from adult to egg, the dengue virus is guaranteed to survive until the next summer and heavy rains. ${ }^{6}$

\section{Japanese encephalitis}

The Japanese encephalitis virus is not found in certain areas; however the mosquito capable of spreading can be present. Japanese encephalitis is a potentially fatal disease and there has been found few reported cases around limited regions. Japanese encephalitis is a disease transmitted by Culex mosquito. It primarily affects birds and animals and accidentally affects humans. The disease causes encephalitis and meningitis, and symptoms include severe headache, fever, stupor, tremors, disorientation, coma, loss of coordination and other meningeal signs. Diagnosis is based on various blood tests and treatment is symptomatic. Pigs are an important maintenance host for this virus, which is mainly transmitted by night-biting mosquitoes in the Culex tritaeniorhynchus group. Thirty percent of those who exhibit encephalitis symptoms die and another 30\% develop serious and permanent neurological damage. Most infections however are asymptomatic and an effective vaccine is available for preventing this infection. $^{7}$

\section{West nile virus}

West Nile virus is a disease transmitted to peoples, horses and birds, and it is the most commonly reported mosquito-transmitted disease around few regions. Most people's infected with West Nile virus show no symptoms or flu-like symptoms, but some (primarily elderly) have more severe illness. West Nile virus is found in some regions and will remain a public health concern in the foreseeable future. ${ }^{8}$

\section{Murray valley encephalitis}

Murray valley encephalitis virus causes symptoms in approximately one in a thousand peoples that is bitten by an infected mosquito. Most persons do not develop any symptoms at all. In adults and older children, symptoms include, fever, drowsiness, bad headache and stiff neck, nausea, muscle tremors and dizziness. In young children the symptoms include fever, floppiness, irritability, drowsiness and fits. The disease can be mild or severe, but in severe cases can lead to coma and death. The incubation time for Murray valley encephalitis varies from 5 to 15 days, but symptoms usually appear within 8 to 10days after being infected. The infection is a greater risk near swamps, floodplains, river systems, irrigation areas and major dams. Peoples with suspected Murray valley encephalitis should be taken to the nearest hospital. ${ }^{9}$

\section{Kunjin virus disease}

This is also a rare similar disease, but generally milder than Murray Valley encephalitis. The virus is also spread by the common banded mosquito. ${ }^{10}$

\section{Ross river virus disease}

This potentially debilitating disease results in rash, fever, swelling and pain in the joints, and is spread by a number of mosquito species including the salt marsh mosquito. ${ }^{11}$

\section{Barmah forest virus disease}

This disease is similar to Ross River virus disease, but is generally milder and less common. It is caused by viruses that are transmitted by the bite of an infected mosquito. ${ }^{12}$

In nature, Murray valley encephalitis, Ross river disease and Barmah forest disease are passed back and forth between wildlife and some species (types) of mosquitoes. Humans can only catch these diseases through the bite of an infected mosquito. The incubation period (the time between being bitten by an infected mosquito and becoming sick) for Ross river and Barmah forest diseases varies from 3 to 21 days, but is normally 7 to 14 days. It may be possible for humans to pass the virus back to mosquitoes that bite them, but only during the incubation period. Peoples living, camping or recreating within $3-5 \mathrm{~km}$ of saltmarshes, estuaries, tidal rivers and freshwater wetlands are at greater risk of Ross river disease and Barmah forest disease infections than peoples living further away. Ross river disease and Barmah forest disease cause symptoms in less than one in three peoples bitten by an infected mosquito. Symptoms vary from person to person, but include painful and swollen joints, sore muscles, aching tendons, skin rashes, fever, tiredness, headaches and swollen lymph nodes. Less common symptoms include, sore eyes, a sore throat, nausea and tingling in the palms of the hands or soles of the feet. Because the symptoms may be similar to some rheumatic diseases or other viral diseases, a specific blood test organized by a doctor is the only reliable method of diagnosis. ${ }^{13}$

\section{La crosse encephalitis}

La Crosse encephalitis is a viral disease that is transmitted by the tree hole mosquitoes. Most peoples are infected by the eastern tree hole mosquito Aedes triseriatus, which is an aggressive daytime biting mosquito commonly found in wooded areas. It is an arthropodborne virus (arbovirus) spread by the bite of infected mosquitoes and infection can lead to severe febrile illness, encephalitis (inflammation of the brain) or meningitis (inflammation of the lining of the brain and spinal cord). While many people's infected have no apparent symptoms, severe disease occurs most often in children under 16 years of age. In rare cases, long-term disability or death can result from this encephalitis. There is no specific treatment for this infection, and care is based on symptoms. Reducing exposure to mosquito bites is the best defense against getting infected with mosquito-borne viruses. ${ }^{14}$ 


\section{Jamestown canyon virus}

Jamestown canyon virus, which may be transmitted by several different species of mosquitoes, is a rarely reported cause of illness in humans. The virus Bunyavirus is closely related to La Crosse virus, although disease is reported less frequently and any age group may be affected. Jamestown canyon virus is an illness that is transmitted to peoples through the bite of an infected mosquito. Symptoms may include fever, headache and flu-like illness. Severe cases involving the central nervous system may include meningitis (inflammation of the membranes surrounding the brain) or encephalitis (inflammation of the brain). Treatment for Jamestown canyon virus involves supportive care until the illness resolves. Several species of mosquitoes are able to transmit virus, including many that commonly bite people and cases of human disease may occur anytime during the warmer months. ${ }^{15}$

\section{Western equine encephalitis}

Western equine encephalitis is a disease transmitted to peoples, horses and birds, and caused by a virus belongs to the genus Alphavirus, in the family Togaviridae. These alphaviruses are spherical and have a diameter of $60-65 \mathrm{~nm}$. The outer layer consists of a glycoprotein shell with protruding glycoprotein spikes, beneath which lies the lipid bilayer. The nucleocapsid core contains the singlestranded ribonucleic acid (RNA) genome. The primary complications other than death in the fever are variable levels of central nervous system impairment. Numerous factors, including location and specific inflammatory cell response, may determine the resulting impairment. Demyelination is a known byproduct of this disease, and it can be detected radiologically. Often, these areas heal quite well unless overlying fibrosis or cell death occurs. Additional complications include mental retardation, behavioral changes, paralysis, permanent focal neurologic deficits, seizure disorders, cerebellar damage and choreoathetosis. Cases of Parkinson syndrome have been reported in adults after equine encephalitis infection. It causes the most severe disease in children $(<15$ years old) and older peoples $(>50$ years old). Those who survive generally suffer permanent, debilitating brain damage. Birds provide the primary source of infection. Several types of daylight or twilight biting mosquitoes transmit it to peoples primarily by the vector mosquito Culex tarsalis. Other mosquitoes (Aedes species) and, occasionally, small, wild mammals also have been known to spread the virus. C. tarsalis is a mosquito that often prefers warm, moist environments. In these locations, cycles of wild bird and mosquito interactions and infectivity allow the virus to remain endemic. No cases of bird transmission of the disease have been reported, making mosquitoes the primary vector and birds simply reservoirs. Epidemic outbreaks in the equine or pheasant population often precede human epidemics of equine encephalitis and no vaccine or curative drug treatment is currently available. ${ }^{16}$

\section{Eastern Equine Encephalitis}

Eastern equine encephalitis is a rare illness in humans and many people's infected with Togaviridae virus show no symptoms, but some (primarily children) have severe illness. Eastern equine encephalitis virus is maintained in a cycle between Culiseta melanura mosquitoes and avian hosts in freshwater hardwood swamps. But, the Cs. melanura is not considered to be an important vector of this to humans because it feeds almost exclusively on birds. Transmission to humans requires mosquito species capable of creating a bridge between infected birds and uninfected mammals, such as the cattail marsh mosquito Coquillettidia perturbans. There have been no reports of human cases contracted; however, the virus has been detected frequently over the years in mosquitoes and birds. In addition, there is a history of horses infected with this disease, including a large equine outbreak. In humans, infection can result in one of two types of illness such as systemic or encephalitic. Systemic infections have an abrupt onset and are characterized by chills, fever, malaise, arthralgia and myalgia. The illness lasts for 1 to 2 weeks and recovery is complete when there is no central nervous system involvement. In infants, the encephalitic form is characterized by abrupt onset, while in older children and adults, encephalitis is manifested after a few days of systemic illness. Signs and symptoms in encephalitic patients include fever, headache, irritability, restlessness, drowsiness, anorexia, vomiting, diarrhea, cyanosis, convulsions and coma. No specific antiviral treatment for infections is available and suspected patients should be hospitalized, appropriate serologic and other diagnostic tests ordered and supportive treatment provided. Approximately one-third of those who develop disease die and many of those who survive can have mild to severe permanent neurologic damage. Many patients with severe sequelae die within a few years. There is no vaccine against it and reducing exposure to mosquito bites is the best defense against getting infected with disease or other mosquito-borne viruses. ${ }^{17}$

\section{Venezuelan equine encephalitis or encephalomyelitis}

Venezuelan equine encephalitis virus is a mosquito-borne viral Togaviridae pathogen that encompasses Venezuelan equine encephalitis or encephalomyelitis. This can affect all equine species, such as horses, donkeys and zebras. After infection, equines may suddenly die or show progressive central nervous system disorders. Humans also can contract this disease wherein healthy adults who become infected by the virus may experience flu-like symptoms, such as high fevers and headaches. Peoples with weakened immune systems and the young or the elderly can become severely ill or die from this disease. The virus is transmitted primarily by mosquitoes that bite an infected animal and then bite and feed on another animal or human. The speed with which the disease spreads depends on the subtype of the virus and the density of mosquito populations. Currently, there is a vaccine available for both humans and horses. The live attenuated vaccine known as TC-83 is a strain of Venezuelan equine encephalitis virus that is passed 83times in guinea pig heart cells. There is also an inactivated form of the vaccine known as $\mathrm{C}-84$ derived from the TC-83 strain. $^{18}$

\section{Wesselsbron fever}

Wesselsbron disease is caused by Wesselsbron virus in the genus Flavivirus of the family Flaviviridae. This virus has the properties typically found in a hemagglutinating flavivirus, but is not yet wellcharacterized. Wesselsbron virus is transmitted by mosquitoes in the genus Aedes including A. caballus and A. circumluteolus. A high seroprevalence among domestic herbivores in warm, moist regions suggests that these animals may have a role as viral reservoirs. In ruminants, there seems to be no direct virus transmission between animals; however, humans have been infected by handling the virus or contaminated material and in these cases, transmission is thought to occur by contact or possibly aerosols. Most infections in humans seem to be subclinical or consist of mild symptoms, such as fever, that are not investigated. In the few symptomatic cases that have been reported, Wesselsbron disease has resembled influenza. The symptoms include fever, headache, arthralgia and muscle pains. Cutaneous hypersensitivity and mild skin rashes have also been reported. Although the fever usually disappears after 2-3days, the muscle pains have been known to persist for much longer. Treatment is symptomatic; there 
is no specific treatment for the virus. For prevention, peoples who work with Wesselsbron virus or contaminated tissues should wear gloves and other protective clothing, and avoid techniques that would aerosolize the virus. Mosquito control measures such as repellants and mosquito netting also decrease the risk of infection. ${ }^{19}$

\section{California encephalitis virus}

Encephalitis is an acute inflammation of the brain that can cause minor symptoms, such as headaches, to more severe symptoms such as seizures. Mosquitoes serve as its carrier and for this reason this virus is known as an arbovirus (arthropod-borne virus). California encephalitis virus belongs to the Bunyaviridae family of viruses and other viruses from the same genus are also a common cause of encephalitis. Other viruses with similar disease symptoms but genetically unrelated include St. Louis encephalitis and West Nile virus. The incubation period of California encephalitis is usually 3-7days. An early symptoms phase of 1-4days commonly precedes the onset of encephalitis. This phase manifests as fever, chills, nausea, vomiting, headache and abdominal pain. ${ }^{20}$

\section{Bwamba fever}

Bwamba fever virus is from the genus Orthobunyavirus and belongs to the family Bunyaviridae. It has a negative sense single stranded RNA (ssRNA) genome, and the genome is segmented into three pieces, Large (L), Medium (M) and Small (S), which have a combined length of approximately 12,000 nt. The S RNA encodes a nucleocapsid and non-structural proteins, the M RNA encodes envelope glycoproteins and a non-structural membrane polypeptide and the L RNA encodes an RNA dependent RNA polymerase. ${ }^{21}$

\section{Bunyamwera}

Hanta virus or Hantavirus Hemorrhagic fever is associated with high fever, lung edema and pulmonary failure and mortality is around $55 \%$. The antibody reaction plays an important role in decreasing levels of viremia. Human infections with certain Bunyaviridae, such as Crimean-Congo hemorrhagic fever virus are associated with high levels of morbidity and mortality, consequently handling of these viruses must occur with a Biosafety level. They are also the cause of severe fever with thrombocytopenia syndrome..$^{22}$

\section{Oropouche fever}

The Oropouche virus is one of the most common orthobunyaviruses. When virus infects humans, it causes a rapid fever illness called Oropouche fever. This is considered to be an arbovirus due to the method of transmission by the mosquitos A. serratus and $C$. quinquefasciatus among sloths, marsupials, primates, and birds. A possible dispersal could be predicted for the four genotypes based on time-scaled analysis and epidemiologic data association. ${ }^{23,24}$

\section{St. Louis encephalitis}

Cases of St. Louis encephalitis are usually the result of unpredictable and intermittent localized epidemics. Saint Louis encephalitis virus is transmitted to humans by the bite of an infected mosquito. Most persons infected with virus have no apparent illness. Initial symptoms of those who become ill include fever, headache, nausea, vomiting and tiredness. Severe neuroinvasive disease (often involving encephalitis, an inflammation of the brain) occurs more commonly in older adults. In rare cases, long-term disability or death can result. There is no specific treatment for the infection; care is based on symptoms. The risk of being infected with virus can be reduced by using insect repellent, wearing protective clothing and staying indoors while mosquitoes are most active. If a person is a family member and may have virus neuroinvasive disease, it is important to consult healthcare provider for proper diagnosis. ${ }^{25}$

\section{Rift valley fever}

Rift Valley fever is an acute, fever-causing viral disease most commonly observed in domesticated animals such as cattle, buffalo, sheep, goats and camels, with the ability to infect and cause illness in humans. The disease is caused by rift valley fever virus, which is a member of the genus Phlebovirus in the family Bunyaviridae. It is transmitted to animals and humans by day-biting Aedes mosquitoes. Humans can also acquire infections through contact with the blood of infected animals during slaughter. Many cases of this are relatively mild, but the hemorrhagic form of this disease has a case fatality rate of over $50 \%$. The overall case fatality rate associated with human infection is probably less than $1 \%$. This fever is one of the few viral pathogens that can be transmitted to the eggs of infected mosquitoes, thus some mosquitoes can be infected before they ever bite a host. No vaccine or curative drug treatment is currently available for it. ${ }^{26}$

\section{Chandipura virus}

Chandipura virus is a member of the family Rhabdoviridae that is associated with an encephalitic illness in humans. Chandipura virus is an enveloped RNA virus with an approximate genome length of $\sim 11 \mathrm{~kb}$. Viral life cycle is cytosolic and during transcription, viral polymerase synthesizes five discrete mRNAs and obeys to stop signals that are present at the gene boundaries. Accumulation of adequate amounts of viral proteins within infected cells through viral transcription and subsequent translation potentiate the onset of viral replicative cycle. In this phase, same L protein acts as a replicase and ignores the gene junctions to generate a polycistronic anti-genomic analogue that acts as a template for further rounds of replication to generate many more copies of the genome RNA. This progeny genome RNA upon packaging by viral proteins buds out as mature virus particles. High grade fever of short duration, vomiting, altered sensorium, generalized convulsions, decerebrate posture, leading to Grade IV coma, acute encephalitis/ encephalopathy, death within a few hours to $48 \mathrm{hrs}$ of hospitalization can occur. The virus earlier has been isolated from sandflies- Phlebotomus species, while successful experimental mouse- mosquito- mouse transmissions have been carried out by six species of mosquitoes A. aegypti, A albopictus, An. stephensi, $C x$. tritaeniorhynchus, $C x$. bitaeniorhynchus and $C x$. Quinquefasciatus. ${ }^{27,28}$

\section{Mosquito prevention and control}

Most often it is performed following the Integrated Mosquito Management (IMM) concept, which is based on ecological, economical and social criteria, and by integrating multidisciplinary methodologies that are practical and effective to protect public health, environment and improve the quality of life. Mosquitoesmanagement can be divided into two areas of responsibilities, the protection from mosquito bites and population controlof mosquitoes to reduce their damage to human health, economies and enjoyment. ${ }^{29}$

\section{Protection from mosquito bites}

There are several approaches to whom adopting a person and family can practice to prevent mosquito-borne diseases.

Avoid mosquito bites: Avoid mosquito bites by escape of outdoor activities at dusk and dawn, the peak feeding time for many mosquitoes or consider avoiding outdoor activities during these times wear, long-sleeved shirts and long pants (light-colored clothing) and use repellents according to label directions. ${ }^{30}$ 
Use insect repellent outdoors: Repellents containing picaridin, and some oil of lemon eucalyptus and para-menthane-diol products provide longer-lasting protection. To optimize safety and effectiveness, repellents should be used according to the label instructions. ${ }^{31}$

Wear long sleeves, pants and socks outdoors: Mosquitoes may bite through thin clothing, so spraying clothes with repellent containing permethrin or another registered repellent can give extra protection. Do not apply repellents containing permethrin directly to skin and do not spray repellent on the skin under clothing. ${ }^{32}$

Care during peak mosquito biting: Take extra care to use repellent and protective clothing from dusk to dawn during peak mosquito biting period and breeding season. ${ }^{33}$

Mosquito proof home: Install or repair screens on windows and doors to keep mosquitoes outside and use air conditioning, if someone have it. It is helpful to reduce the number of mosquitoes around home by emptying standing water from flowerpots, gutters, buckets, pool covers, pet water dishes, discarded tires and birdbaths on a regular basis. $^{34}$

\section{Mosquitos population control programs}

It is helpful to support local community mosquito control programs because vector control activities are most often handled at the local level, such as through county or city government. The type of mosquito control methods used by a program depends on the time of year, the type of mosquitoes to be controlled and the habitat structure. Methods can include elimination of mosquito larval habitats, application of insecticides to kill mosquito larvae or spraying insecticides from trucks or aircraft to kill adult mosquitoes. Local mosquito control program can provide information about the type of products being used in an area. Inform about the dead birds to local authorities because dead birds may be a sign that West Nile virus and other arboviruses are circulating between birds and the mosquitoes in an area. By reporting dead birds or animals to local health departments, anyone can play an important role in monitoring mosquito-borne diseases. Local agencies have different policies for collecting and testing birds, so check with local health department to find information about reporting dead birds in an area. ${ }^{35-37}$

Remove or regularly clean sources of standing water from possessions where mosquitoes can breed, remove or turn over any containers that could hold water (tires, cans, pet dishes, toys, cans, flower pot saucers) and check land in the spring before plants have the chance to grow and hide these objects. Change the water in bird baths at least once a week to prevent mosquito breeding and recycle old tires or store them where they cannot collect rainwater. If these are not options, then cover them with a tight tarp or store them in direct sunlight. Check gutters and clean out frequently to make sure they are not plugged. Fill water-holding tree holes with dirt or sand to prevent further mosquito breeding. For protecting of ourselves from mosquito-borne diseases, limit outdoor activity after dark during peak mosquito season. Avoid moving in known mosquito-infested areas (e.g., swamps, marshes) during those times as well. When anyone is outdoors after dark during mosquito season, or when camping out, sleep under mosquito nets or use insecticide treated nets. ${ }^{38,39}$

Under certain circumstance, the continuous application of synthetic insecticides causes development of resistance in vector species, biological magnification of toxic substances through the food chain and adverse effects on environmental quality ${ }^{40-42}$ and non-target organisms including human, wildlife and aquatic health issues. ${ }^{43-44}$ Application of active toxic agents from plant extracts as an alternative mosquito control strategy, which are non-toxic, easily available at affordable prices, biodegradable and show broad-spectrum targetspecific activities against different species of vector mosquitoes. Extracts of several plants such as neem (Azadirachta indica), Peppermint (Mentha piperata), lemon eucalyptus (Corymbiach citriodora) and basil (Ocimum basilicum) have good repellents efficacy against some mosquito species. ${ }^{45,46}$

\section{Conclusion}

Mosquitoes are found throughout the world and many species transmit pathogens which may cause viral diseases. These diseases include mosquito-borne viral encephalitis, dengue, yellow fever and filariasis. Most of these diseases have been prominent as endemic or epidemic diseases in the various states in the past, but today, only the insect-borne (arboviral) encephalitides occur annually and dengue occurs periodically. The major types of viral encephalitis include St. Louis, La Crosse, Eastern equine and Western equine. These viruses are normally infections of birds or small mammals. During such infections, the level of the virus may increase in these infected animals, thus facilitating transmission to humans by mosquitoes. The West Nile virus, which can also cause encephalitis, is a good example of this mode of transmission. Human cases of encephalitis range from mild to very severe illnesses that in a few cases can be fatal. Control measures could be aimed at different stages of the mosquito in its life cycle. The measures can be classified as biological, environmental and chemical control. Integrated mosquito management approach should be adopted for getting an effective and efficient control on mosquito with minimal impact on the ecological system.

\section{Acknowledgments}

None.

\section{Conflicts of interest}

Author declares there are no conflicts of interest.

\section{Funding}

None.

\section{References}

1. Vasconcelos PEC, Travassos Da Rosa MA, Degallier N, et al. Clinical and ecoepidemiological situation of human arboviruses in Brazilian Amazonia. Journal of the Brazilian Association for the Advancement of Science. 1992;44 (2/3):117-124.

2. Sarwar M. Reducing Dengue Fever through Biological Control of Disease Carrier Aedes Mosquitoes (Diptera: Culicidae). International Journal of Preventive Medicine Research. 2015;1(3):161-166.

3. Sarwar M. Elimination of Dengue by Control of Aedes Vector Mosquitoes (Diptera: Culicidae) Utilizing Copepods (Copepoda: Cyclopidae). International Journal of Bioinformatics and Biomedical Engineering. 2015;1(1):53-58.

4. Gratz NG. Critical review of the vector status of Aedes albopictus. Med Vet Entomol. 2004;18:215-227.

5. Duffy MR, Chen TH, Hancock WT, et al. Zika virus outbreak on Yap Island, Federated States of Micronesia. $N$ Engl $J$ Med. 2009;360(24):2536-2543.

6. Sarwar M. Defeating Malaria with Preventative Treatment of Disease and Deterrent Measures against Anopheline Vectors (Diptera: Culicidae). Journal of Pharmacology and Toxicological Studies. 2014;2(4):1-6.

7. Tiroumourougane SV, Raghava P, Srinivasan S. Japanese viral encephalitis. Postgraduate Medical Journal . 2002;78(918):205-215. 
8. Hector C. Emergency Department Management of Mosquito-Borne Illness: Malaria, Dengue, and West Nile Virus. Emerg Med Pract. 2014;16(5):1-23.

9. Calisher CH, Gould EA. Taxonomy of the virus family. In: Maramorosch $\mathrm{K}$, et al. (Eds.). Advances in virus research. Academic Press, San Diego. 2003. p. $1-19$.

10. Sarwar M . Proposing Solutions for the Control of Dengue Fever Virus Carrying Mosquitoes (Diptera: Culicidae) Aedes aegypti(Linnaeus) and Aedes albopictus(Skuse). Journal of Pharmacology and Toxicological Studies. 2014;2(1):1-6.

11. Sarwar M . Dengue Fever as a Continuing Threat in Tropical and Subtropical Regions around the World and Strategy for Its Control and Prevention. Journal of Pharmacology and Toxicological Studies . 2014;2(2):1-6.

12. Sarwar M. Proposals for the Control of Principal Dengue Fever Virus Transmitter Aedes aegypti (Linnaeus) Mosquito (Diptera: Culicidae). Journal of Ecology and Environmental Sciences . 2014;2(2):24-28.

13. Lounibos LP. Invasions by insect vectors of human disease. Annu Rev Entomol. 2002;47:233-166.

14. Vasconcelos PFC, Travassos da Rosa APA, Pinheiro FP, et al. Arboviroses. In: Focaccia R, et al. (Eds.), Tratado de infectologia. Sao Paulo: Atheneu. 2005;1:289-302.

15. Bezirtzoglou C, Dekas K, Charvalos E. Climate changes, environment and infection: facts, scenarios and growing awareness from the public health community within Europe. Anaerobe . 2011;17(6):337-340.

16. Gubler DJ, Kuno G, Markoff L. Flaviviruses. (5th edn), In: Knipe DM \& Howley PM (Eds.), Fields virology. Philadelphia: Lippincott Williams \& Wilkins, USA. 2007. p.1153-1252.

17. Verwoerd DJ. Ostrich diseases. Rev Sci Tech. 2000;19(2):638-661.

18. Auguste AJ, Volk SM, Arrigo NC, et al. Isolation and phylogenetic analysis of Mucambo virus (Venezuelan equine encephalitis complex subtype IIIA) in Trinidad. Virology. 2009;392(1):123-130.

19. Mushi EZ, Binta MG, Raborokgwe M. Wesselsbron disease virus associated with abortions in goats in Botswana. $J$ Vet Diagn Invest . 1998;10(2):191.

20. Eldridge BF, Glaser C, Pedrin RE, et al. The First Reported Case of California Encephalitis in More Than 50 Years. Emerg Infect Dis. 2001; 7(3): 451-452.

21. Aultman KS, Beaty BJ, Walker ED. Genetically manipulated vectors of human disease: a practical overview. Trends Parasitol. 2001;17(11):507-509.

22. Figueiredo LT. The Brazilian flaviviruses. Microbes Infect. 2000;2(13):1643-1649.

23. Vasconcelos HB, Nunes MRT, Casseb LMN, et al. Molecular Epidemiology of Oropouche Virus, Brazil. Emerging Infectious Diseases. 2011;17(5):800-806.

24. Saeed MF, Wang $\mathrm{H}$, Nunes $\mathrm{M}$, et al. Nucleotide sequences and phylogeny of the nuclecapsid gene of Oropouche virus. J Gen Virol. 2000;81(3):743-748.

25. Reisen WK. Epidemiology of St. Louis encephalitis virus. Adv Virus Res. 2003;61:139-183.

26. Jensen T, Cockburn AF, Kaiser PE, et al. Human blood-feeding rates among sympatric sibling species of Anopheles quadrimaculatus mosquitoes in northern Florida. Am J Trop Med Hyg. 1996;54(5):523-525.

27. Basak S, Mondal A, Polley S, et al. Reviewing Chandipura: A Vesiculovirus in Human Epidemics. Biosci Rep. 2007;27(4-5):275-298.

28. Chadha MS, Arankalle VA, Jadi RS, et al. An Outbreak of Chandipura Virus Encephalitis in the Eastern Districts of Gujarat State, India. Am J Trop Med Hyg . 2005;73(3):566-570.
29. Sarwar M. Control of Dengue Carrier Aedes Mosquitoes (Diptera: Culicidae) Larvae by Larvivorous Fishes and Putting It into Practice Within Water Bodies. International Journal of Preventive Medicine Research. 2015;1(4):232-237.

30. Sarwar M. Controlling Dengue Spreading Aedes Mosquitoes (Diptera: Culicidae) Using Ecological Services by Frogs, Toads and Tadpoles (Anura) as Predators. American Journal of Clinical Neurology and Neurosurgery. 2015;1(1):18-24.

31. Lutwama JJ, Rwaguma EB, Nawanga PL, et al. Isolations of Bwamba virus from south central Uganda and north eastern Tanzania. Afr Health Sci. 2002;2(1):24-28.

32. Sarwar M. Role of Secondary Dengue Vector Mosquito Aedes albopictus Skuse (Diptera: Culicidae) for Dengue Virus Transmission and Its Coping. International Journal of Animal Biology. 2015;1(5):219-224.

33. Sarwar M. Families of Common Synthetic Agrochemicals Designed to Target Insect Pests or Vectors in Landscapes and Households. Chemistry Research Journal. 2016;1(3):7-13.

34. Sarwar M. Source Reduction Practices for Mosquitoes (Diptera) Management to Prevent Dengue, Malaria and Other Arboborne Diseases. American Journal of Clinical Neurology and Neurosurgery. 2015;1(2):110-116.

35. Sarwar M . Intervention Focused on Habitat Modifications for Ending up the Anopheles Mosquitoes Implicating in Malaria Transmission. American Journal of Clinical Neurology and Neurosurgery. 2015;1(2):126-132.

36. Sarwar M. Indoor risks of pesticide uses are significantly linked to hazards of the family members. Cogent Medicine. 2016;3:1155373.

37. Sarwar M. Stopping Breeding of Dengue Virus Spreader Aedes Mosquitoes (Diptera: Culicidae) with Environmental Modifications. International Journal of Bioinformatics and Biomedical Engineering. 2015;1(2):169-174.

38. Sarwar M. Usage spots of biological insecticides in consort with target insect pests or vectors and application in habitat. International Journal of Entomology and Nematology. 2016;3(1):14-20.

39. Sarwar M. Inorganic Insecticides used in Landscape Settings and Insect Pests. Chemistry Research Journal. 2016;1(1):50-57.

40. Sarwar M. Potential Uses of Synergists in Insecticides Resistance Management Accompanied by Their Contributions as Control Agents and Research Tools. Chemistry Research Journal . 2016;1(3):21-26.

41. Sarwar M. Outdoors Agricultural Insecticides Pose worth Global Risks and Espousal of Safety Practices among Farmers. International Journal for Research in Agricultural Research. 2016;1(7):1-9.

42. Sarwar M. A Glance at Pesticides Usage: Remunerations and Complications Associated with Insecticides Putting in Practice. International Journal for Research in Agricultural Research . 2016;1(7):10-19.

43. Sarwar M. Insecticide Risk Exposes Threat to Aquatic Life in Surface Water Bodies and its Remedying. International Journal for Research in Applied Chemistry. 2016;1(6):1-9.

44. Sarwar M. Pesticide's Dangers to Wildlife and Safeguard Dealings in Reducing of the Risks. International Journal for Research in Health Sciences and Nursing. 2016;1(7):15-25.

45. Nicoletti M, Murugan K, Serrone PD. Current mosquito-borne disease emergencies in Italy and climate changes. The neem opportunity. Trends in Vector Research and Parasitology. 2014; 1:1-9.

46. Sarwar M. A potent folklore of botanical plant materials against insect pests together with their preparations and applications. Sky Journal of Biochemistry Research. 2016;5(4):58-62. 